American Journal of Nanotechnology 1 (1): 1-6, 2010

ISSN 1949-0216

(C) 2010 Science Publications

\title{
Modeling of Piezoelectric Actuators Based on Bayesian Regularization Back Propagation Neural Network
}

\author{
Wen Wang, Zhu Zhu, Yanding Wei and Zichen Chen \\ Department of Mechanical Engineering, Zhejiang University, \\ Hangzhou 310027, P.R. China
}

\begin{abstract}
Problem statement: Piezoelectric actuator is a kind of key driving components for micropositioning stages, micropumps, micro valves, micro gripper and so on in the fields of micro/nano technology such as integrated circuit manufacturing, precision instruments, ultra precision fabrication, biomedical manipulation. It has lots of advantages including high stiffness, fast response times, less heat generating, low power consumption and large force output. But the hysteresis nonlinearity seriously affects working performance of actuators. So a lot of models were proposed to describe the hysteresis nonlinearity. A popular model which was widely used is the Preisach model. In order to obtain accurate displacement output corresponding to arbitrary input voltage with the Preisach model, function output approximation is needed. Approach: In this study, firstly the Preisach model was introduced. Then the function modeling of Preisach model based on a Bayesian Regularization Back Propagation Neural (BRBPNN) was presented and a three layers BPNN was designed. Finally, the BRBPNN was trained in Neural Network toolbox of MATLAB6.0. The Preisach function values not at equal diversion points were calculated by the trained network and the actual displacement outputs and theoretical values corresponding to random voltages input were compared. Results: Experimental results indicate that theoretical displacements and measured displacements agree with very well, the maximum displacement error is $0.35 \mu \mathrm{m}$ and the standard deviation is $0.24 \mu \mathrm{m}$. Conclusion: The BRBPNN could realize function approximation in Preisach modeling accurately and could meet the precision requirement in the field of modeling and controlling of piezoelectric actuators.
\end{abstract}

Key words: Piezoelectric actuators, hysteresis nonlinearity, Bayesian regularization, precision instruments, neural network

\section{INTRODUCTION}

Piezoelectric actuators are widely used in ultraprecision machining, integrated circuit manufacturing, precision instruments (Wang et al., 2010) due to advantages of high displacement resolution, fast response, high stiffness, small volume and less heat generation. But the hysteresis nonlinearity affects their working performance seriously. So modeling for hysteresis nonlinearity is needed (Han et al., 2000; Gruenbichler et al., 2008; Li et al., 2007; Mordjaoui et al., 2007). The Preisach model is a hysteresis model describing the static nonlinearity of piezoelectric actuators, which was proposed by Preisach (1935) based on magnetic effect (Ping and Musa, 1997). When using Preisach model to compute displacement output of a piezoelectric actuator, the density level of the equal diversion points of voltage directly affects the output accuracy. The model has a relatively great error when the number of equal diversion points are relatively less, but as the increasing of equal diversion points, the amount of experimental data which needed to be acquired will grow dramatically. Therefor, how to predict Preisach function value not at equal diversion points by using small amount of measuring points through function output approximation is very important. Preisach model could obtain a relevant displacement output corresponding to an arbitrary voltage input by function approximation. It could reduce the influence of hysteresis and effectively improve the control precision of the piezoelectric actuator.

The traditional method of function approximation includes Bilinear Interpolation, Polynomial Fitting but the approximation accuracy is not high enough for

Corresponding Author: Wen Wang, Department of Mechanical Engineering, Zhejiang University, Hangzhou 310027, P.R. China Fax: +86-571-87951906 
some nonlinear function and these methods do not have self-learning and adaptive capacity, so the application in Preisach modeling is in a certain limit. The modern developed methods, such as Back Propagation Neural Network (BPNN), make up the deficiency of the traditional methods and has broad prospects. The BPNN is a multilayer feed forward neural network. The network and its variations are mostly used in function approximation, pattern recognition, classification, intelligent control. But standard BP algorithm has problems of relevant to the order of the input sample, poor generalization ability, easy to form local minima and slow convergence speed. The improved methods include additional momentum, adaptive learning rate method, Bayesian Regularization algorithm and so on.

Some researchers used neural network to model the hysteresis curve directly. They used different neural networks to fit ascending and descending curves respectively, but when the change history of the input voltage is different from one of the experimental data used in training, the output cannot be predicted by the trained neural networks (Hwang et al., 2001). Some academics also applied neural networks in parameter identification of Preisach model and improved Preisach model. Adly and Abd-El-Hafiz (1998) used the similarity of Preisach model and neural network to achieve the nonlinear and hysteresis modeling of materials without wiping-out property. Liu et al. (2001) used BP and RBF neural network to identify the key parameters of a new kind of generalized Preisach model and realize a high accuracy.

This study utilizes BPNN to realize function approximation based on Preisach model and select Bayesian Regularization method to optimize, which has a marked effect on enhancing generalization ability of network and increasing training speed and has been used in some research works (Aggarwal et al., 2005). The network was trained in Neural Network toolbox of MATLAB6.0 and validated by experiments.

\section{MATERIALS AND METHODS}

Materials: The experiment has been done with a piezoelectric actuator, the WTDS-IA of Sichan Institute of Piezoelectric and Acoustooptic Technology, whose voltage range is $0 \sim 300$ Volts and the maximum displacement output is $20 \mu \mathrm{m}$. A DGS-6C Model digital inductance style micrometer with a resolution of 0.01 $\mu \mathrm{m}$ was used in displacement measurement.

Preisach model methods: Preisach model is piled up by the simplest hysteresis generator and the mathematical expression is (Ping and Musa, 1997): $x(t)=\iint_{\alpha \geq \beta} \mu(\alpha, \beta) \gamma_{\alpha \beta}[\mathrm{u}(\mathrm{t})] \mathrm{d} \alpha \mathrm{d} \beta$

Where:

$\mathrm{x}(\mathrm{t})=$ Output displacement of piezoelectric actuator

$\mathrm{u}(\mathrm{t})=$ Input voltage

$\mu(\alpha, \beta)=$ Weighting function

$\gamma_{\alpha \beta}=$ Elementary rectangular hysteresis operator as shown in Fig. 1

As the input voltage raises from 0 up to $\alpha$ and drops down to $\beta$, the shrinkage of piezoelectric actuator is defined as Preisach function:

$X\left(\alpha^{\prime}, \beta^{\prime}\right)=x_{\alpha^{\prime}}-x_{a^{\prime} \beta^{\prime}}$

Where:

$\mathrm{x}_{\alpha}=$ The output displacement of actuator when the input voltage raises from 0 up to $\alpha$

$\mathrm{X}_{\alpha^{\prime} \beta^{\prime}},=$ Output displacement of actuator when the input voltage drops from $\alpha$ to $\beta$

To avoid the complexity of two partial derivatives and double integrals, Preisach model is discreted. When $\mathrm{u}(\mathrm{t})$ is on the upswing and the decline, the model is Eq. 3-4, respectively:

$$
\begin{aligned}
& x(t)=\sum_{k=1}^{n-1}\left[x\left(\alpha_{k}, \beta_{k-1}\right)-x\left(\alpha_{k}, \beta_{k}\right)\right]+x\left(u(t), \beta_{n-1}\right) \\
& x^{\prime}(t)=\sum_{k=1}^{n-1}\left[x\left(\alpha_{k}, \beta_{k-1}\right)-x\left(\alpha_{k}, \beta_{k}\right)\right]+\left[x\left(\alpha_{n}, \beta_{n-1}\right)\right. \\
& \left.-x\left(\alpha_{n}, u(t)\right)\right]
\end{aligned}
$$

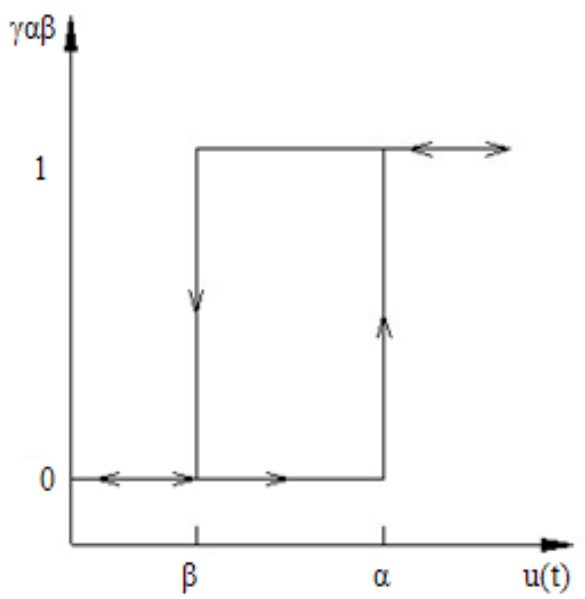

Fig. 1: The picture of hysteresis operator 
Am. J. Nanotech., 1 (1): 1-6, 2010

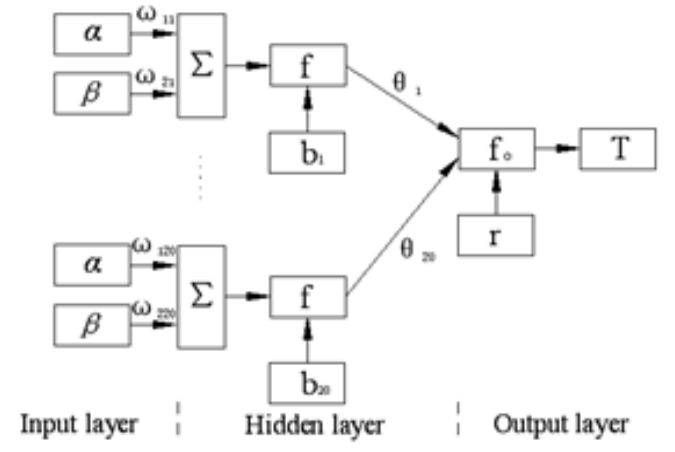

Fig. 2: Framework of back propagation neural network

The table of Preisach function values $X(\alpha, \beta)$ could be obtained by dividing the range of 0 to saturation voltage into $\mathrm{n}$ equal diversions and measuring voltage values at equal diversion points according to the request of Preisach function. In application, for different input voltages, the output displacements could be obtained by substituting Eq. 3-4 the values found in the table of Preisach function values $\mathrm{X}(\alpha, \beta)$.

In order to obtain a relevant accurate displacement output corresponding to an arbitrary voltage input with the Preisach model, BPNN is used in function approximation, that is to predict Preisach function values which is not at equal diversion points by using the small amount of measuring data.

Bayesian regularization algorithm methods: Generalization refers to the ability of neural network reflecting testing samples or working samples correctly after learning, which is an important index of neural network's performance. Regularization algorithm means the process of improve the generalization ability by amending the performance function.

The performance function of standard BP algorithm usually use the mean square error function (Song et al., 2008) mse:

$$
\text { mse }=\sum_{i=1}^{N}\left(t_{i}-a_{i}\right)^{2} / N
$$

Where:

$\mathrm{t}_{\mathrm{i}}=$ Target value

$\mathrm{a}_{\mathrm{i}}=$ Output value

In the regularization method, the network performance function is improved into the following form:

msereg $=\mathrm{p} \times \mathrm{mse}+\mathrm{q} \times \mathrm{msw}$

Where:

$\mathrm{p}$ and $\mathrm{q}=\quad$ Proportional coefficient msw = Average of all network weights' square sum, that is:

$\mathrm{msw}=\sum_{\mathrm{j}=1}^{\mathrm{N}} \mathrm{W}_{\mathrm{j}}^{2} / \mathrm{N}$

If $\mathrm{p}<<\mathrm{q}$, the network focuses on decreasing training error, but over-fitting may occur. If $\mathrm{q}<<\mathrm{p}$, the network focuses on limiting the scale of weights, but errors may be larger. Conventional regularization method is often difficult to determine the size of the proportional coefficient $\mathrm{p}$ and $\mathrm{q}$, but Bayesian Regularization method can adaptively adjust the size of $p$ and $q$ in the training process and optimize the network. In the Bayesian framework, network weights are considered as random variables, the noise and weight vectors exist in sample data obeys Gaussian distribution. Based on Bayesian Regularization criteria, the optimal solution of $p$ and $q$ could be obtained by maximizing the posterior probability when the performance function is of minimum value.

Through the new performance index function, the network could have small weights when the network error be ensured to be as small as possible, which in fact is equivalent to the reducing of the size of network automatically. The shrinkage of the network size (usually referring to the number of hidden layers) could make neural networks' size be much smaller than the size of the training sample, when the training sample is of a certain size, so the probability of over-training is very small, which helps to improve the generalization ability of the network.

The methods of the establishment of BPNN: A BPNN consists of input layer, hidden layer and output layer. Nodes in front layer and back layer are connected by network connection weights and there is no coupling between nodes in the same layer. The learning and training process consists of two parts, that is the forward transmission of the input signal of network and the back transmission of error signal. Robert HechtNielson has proved that any continuous function in an enclosured domain can be fitted by BPNN with one hidden layer (Cybenko, 1989).

A three layers BPNN was designed as shown in Fig. 2. Input layer has two nodes, the rising voltage $\alpha$ and dropping voltage $\beta$. There is one hidden layer, which has 20 neurons. Output layer has one node, the displacement $x_{\alpha \beta}$ corresponding to extreme voltage points. $\omega_{\mathrm{ij}}$ is network weights between the input nodes and hidden nodes. $\theta_{\mathrm{i}}$ is network weights between the hidden nodes and output nodes. 
Am. J. Nanotech., 1 (1): 1-6, 2010

Table 1: Table of Preisach function $\mathrm{X}(\alpha, \beta)$ value (Unit: $\mu \mathrm{m})$

\begin{tabular}{|c|c|c|c|c|c|c|c|c|c|c|}
\hline$\alpha(\mathrm{V})$ & -------ינ & 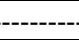 & $\cdots$ & -- & - & 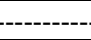 & ---- & 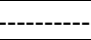 & - & 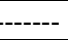 \\
\hline & 0.00 & 30.00 & 60.00 & 90.00 & 120.00 & 150.00 & 180.00 & 210.00 & 240.00 & 270 \\
\hline 300 & 19.98 & 19.40 & 18.25 & 16.29 & 13.72 & 10.76 & 7.95 & 5.46 & 3.33 & 1.54 \\
\hline 270 & 18.13 & 17.36 & 16.22 & 14.32 & 11.81 & 8.90 & 6.11 & 3.69 & 1.67 & \\
\hline 240 & 15.75 & 14.98 & 13.85 & 11.97 & 9.50 & 6.66 & 3.96 & 1.78 & & \\
\hline 210 & 12.86 & 12.09 & 10.97 & 9.13 & 6.72 & 4.11 & 1.78 & & & \\
\hline 180 & 9.69 & 8.92 & 7.80 & 5.96 & 3.78 & 1.59 & & & & \\
\hline 150 & 6.82 & 6.06 & 4.95 & 3.25 & 1.42 & & & & & \\
\hline 120 & 4.34 & 3.60 & 2.54 & 1.24 & & & & & & \\
\hline 90 & 2.46 & 1.77 & 0.85 & & & & & & & \\
\hline 60 & 1.22 & 0.58 & & & & & & & & \\
\hline 30 & 0.57 & & & & & & & & & \\
\hline
\end{tabular}

Table 2: Comparison between measured displacement and theoretical displacement

\begin{tabular}{lccc}
\hline Voltage $(\mathrm{V})$ & Meas. disp. $(\mu \mathrm{m})$ & Theoretical Disp. $(\mu \mathrm{m})$ & Error $(\mu \mathrm{m})$ \\
\hline 0 & 0.00 & 0.00 & 0.00 \\
285 & 19.10 & 19.11 & -0.01 \\
40 & 1.12 & 1.01 & 0.11 \\
100 & 3.34 & 3.11 & 0.23 \\
250 & 16.22 & 16.48 & -0.26 \\
170 & 10.68 & 10.88 & -0.20 \\
110 & 5.02 & 5.27 & -0.25 \\
70 & 2.42 & 2.32 & 0.10 \\
130 & 5.47 & 5.12 & 0.35 \\
160 & 7.92 & 7.58 & 0.34 \\
\hline
\end{tabular}

The network input vector is:

$\mathrm{V}=(\alpha, \beta)^{\mathrm{T}}$

The output of network's hidden layer is:

$y_{j}=f\left(\sum_{i=1}^{2} \omega_{i j} x_{i}-b_{j}\right)$

Where:

$b_{j}=$ The threshold of hidden layer nodes

$\mathrm{f}=$ The activation function of hidden layer, which is sigmoid function and the expression is:

$f(v)=\frac{1}{1+e^{-v}}$

The output of network's output layer is:

$$
\mathrm{T}=\mathrm{f}_{\mathrm{O}}\left(\sum_{\mathrm{j}=1}^{20} \theta_{\mathrm{j}} \mathrm{y}_{\mathrm{j}}-\mathrm{r}\right)
$$

Where:

$r=$ The threshold of output nodes

$f_{o}=$ The activation function of output layer, which is linear function and the expression is:

$f_{O}(x)=k x(k \neq 0)$
The training procedure of BPNN is as follows:

- Initialize the thresholds and weights of the network and set all of them to be small random numbers

- Provide training samples, which is the measured voltage at equal diversion points $(\alpha, \beta)$ and the corresponding Preisach function value $\mathrm{X}(\alpha, \beta)$

- Compute the actual output, the error of hidden layer and output layer using Eq. 8-9 and Eq. 6.

- Adjust the weights until the error is less than the target error

When the network training is completed, fixed weights and thresholds could be getten, then the network has the capacity of function approximation. Input the voltage values which is not at equal diversion points into the network, the corresponding Preisach function value can be received.

Simulation and experiment methods: The piezoelectric actuator's input voltage range of $0 \sim 300 \mathrm{~V}$ was divide into 10 equal diversions. During the experiment, the applying voltage changed from $0-30 \mathrm{~V}$ while the output displacement was recorded, then the applying voltage changed to $0 \mathrm{~V}$ while the output displacement was recorded too. The value of $X(30,0)$ can be calculated out by the two records. Then the applying voltage changed from $0-60 \mathrm{~V}$ and then returned to $30 \mathrm{~V}$ while both output displacements were recorded and the value of $X(60,30)$ can be calculated out. According to this method, the table of Preisach function value $\mathrm{X}(\alpha, \beta)$ has been obtained as Table 1 (Wei and Tao, 2004).

The Neural Network toolbox in MATLAB6.0 was used to train the network. Bayesian Regularization algorithm, hidden layer activation function and output layer activation function were realized by built-in function trainbr, tansig and purelin. The training sample is the experimental data in Table 1.

The main network training parameters are set as follows: the minimum expected error is $0.01 \mu \mathrm{m}$; the revised weights learning rate is 0.1 ; the maximum training cycles is 1,000,000. After 673,004 times training, the expected error is reduced to $0.01 \mu \mathrm{m}$. 
Am. J. Nanotech., 1 (1): 1-6, 2010

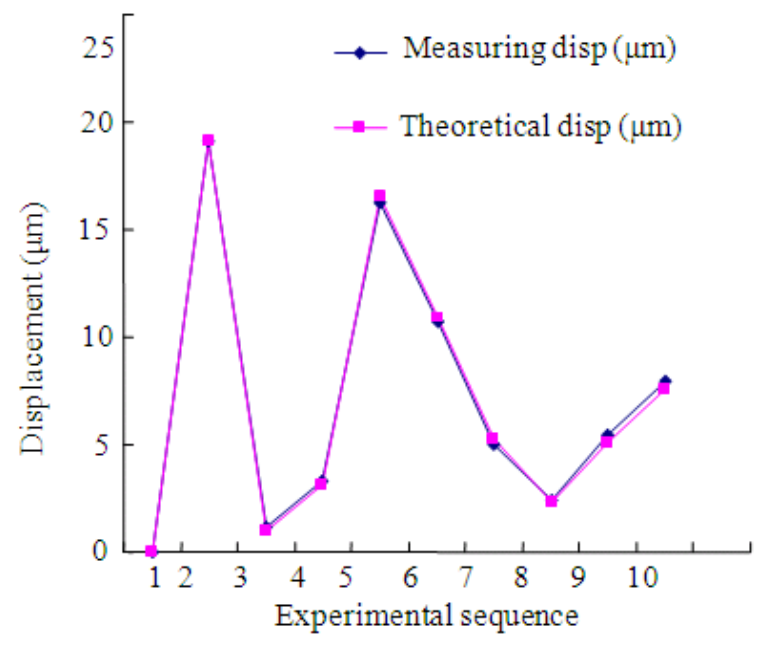

Fig. 3: Comparison between measured curve and theoretical curve of displacement

\section{RESULTS AND DISCUSSION}

In order to verify the fitting performance of BRBPNN, the Preisach function values which is not at the equal diversion points is predicted using the trained network. The theoretical displacement outputs and measuring values corresponding to random voltages input were compared. As shown in Table 2 and Fig. 3, the maximum displacement error is $0.35 \mu \mathrm{m}$ and the standard deviation is $0.24 \mu \mathrm{m}$.

\section{CONCLUSION}

According to the demand of output function approximation in piezoelectric actuator's Preisach model, a Bayesian Regularization algorithm was used to amend the performance function to enhance the neural network's generalization ability. A three layers BPNN has been designed and the sample is the measured data in the table of Preisach function value $\mathrm{X}$ $(\alpha, \beta)$. The BRBPNN was trained in Neural Network toolbox of MATLAB6.0 and which was used to predict displacement outputs corresponding to random voltages input. The results indicate that the theoretical values and measured ones agree with very well.

Through BPNN realizing output function approximation in Preisach model of piezoelectric actuator, Preisach model can be applied in open loop control or feedforward control system to compensate for the inherent hysteresis of piezoelectric actuator and effectively improve the control accuracy.

\section{ACKNOWLEDGMENT}

The researchers would like to thank National Natural Science Foundation of China (Grant No.50875241) and Zhejiang Provincial Natural Science Foundation of China (Grant No.Z1090590)

\section{REFERENCES}

Adly, A.A. and S.K. Abd-El-Hafiz, 1998. Using neural networks in the identification of preisach-type hysteresis models. IEEE/ASME Trans. Mechatron., 34: 629-635. DOI: 10.1109/20.668057

Aggarwal, K.K., Y. Singh, P. Chandra and M. Puri, 2005. Bayesian Regularization in a Neural Network Model to Estimate Lines of Code Using Function Points. J. Comput. Sci., 1: 505-509. DOI: 10.3844/jessp.2005.505.509

Cybenko, G., 1989. Approximation by superpositions of a sigth control. Sign. Syst., 2: 303-314. DOI: CNKI:SUN:XNJT.0.2008-06-009

Gruenbichler, H., J. Kreith and R. Bermejo, 2008. Investigation of the behavior of multilayer piezoelectric actuators: Modeling and experiments. Proceedings of the ASME Conference on Smart Materials, Adaptive Structures and Intelligent Systems, $\quad$ pp: 349-356. http://apps.isiknowledge.com/summary.do?qid=1\& product $=$ WOS $\&$ SID $=1 \mathrm{BcpEoDIOGII} \% 40 \mathrm{aMe} 4 \mathrm{JG}$ \&search_mode $=$ GeneralSearch

Han, J.M., L.K. Willem and B. Reinder, 2000. Modeling piezoelectric actuators: IEEE/ASME Trans. Mechatron., 5: 331-341. DOI: $10.1109 / 3516.891044$

Hwang, C.L., C. Jan and Y.H. Chen, 2001. Piezomechanics using intelligent variable-structure control. IEEE Trans. Indus. Elect., 48: 47-59. DOI: 10.1109/41.904550

Li, X., W. Wang, J. Chen and Z. Chen, 2007, Hysteresis compensation of giant magnetostrictive actuator based on Jiles-Atherton model. Optics Precision Eng., 10:1558-1563. DOI: CNKI:SUN:GXJM.0.2007-10-018

Liu, X.D., Y. Liu and L. Li, 2001. A new kind of generalized Preisach hysteresis model and its identification based on neural network. Trans. Beijing Inst. Technol., 27: 135-138. DOI: CNKI:ISSN:1001-0645.0.2007-02-009

Mordjaoui, M., M. Chabane, B. Boudjema and R. Daira, 2007. qualitative ferromagnetic hysteresis modeling. J. Comput. Sci., 3: 399-405. DOI: $10.3844 /$ jcssp.2007.399.405 
Ping, G. and J. Musa, 1997. Generalized Preisach model for hysteresis nonlinearity of piezoceramic actuators. Precision Eng., 20: 99-111. DOI: 10.1016/S0141-6359(97)00014-7

Song, L., T. Hang and J. Fang, 2008. Conversion of GPS height based on Bayesian regularization BP neural network. J. Southwest Jiaotong Univ., 43: 724-728. DOI: CNKI:SUN:XNJT.0.2008-06-009
Wei, Y. and H. Tao, 2004, Study on the preisach model of hyteresis in piezoelectric actuator. Piezoelectrics Acoustooptics, 5: 346-367. DOI: CNKI:SUN:YDSG.0.2004-05-006

Wang, W., Z. Zhu and Z. Chen, 2010, Design and analysis of a $2 \mathrm{D} X-Y$ micropositioning stage. Proc. SPIE, 7544: 75441I. DOI: 10.1117/12.885293 